REOP. Vol. 11, No 20, $2^{\circ}$ Semestre, 2000, pp. 229-240

\title{
ORIENTACIONES PARA HACER VIABLES LAS ESTRATEGIAS DE ADAPTACIÓN EN EDUCACIÓN SECUNDARIA OBLIGATORIA
}

\author{
MAKING ADAPTIVE EDUCATION STRATEGIES FEASIBLE \\ AT THE SECONDARY SCHOOLS
}

\author{
Mercedes García García* \\ Universidad Complutense de Madrid
}

\section{RESUMEN}

Este artículo defiende las situaciones en las que se debe abordar la adaptación educativa y justifica los elementos que hacen posible el desarrollo de un proyecto global de atención a la diversidad en la escuela. A partir de las teorías adaptativas y de las reflexiones surgidas tras la evaluación de las estrategias de adaptación implementadas en un centro de secundaria de la periferia de Madrid se plantea la necesidad de: un marco teórico, una cultura de atención a la diversidad, un trabajo cooperativo coordinado por el departamento de orientación y un apoyo de la administración educativa.

Palabras clave: Estrategias de adaptación educativa. Educación Secundaria Obligatoria. Viabilidad.

\begin{abstract}
This article tries to analyse the components that we must introduce in schools in order to plan and develop an educational project focused on teaching adapted to individual differences. The author formulates her own reflections on adaptive education theory and from the evaluation of adaptive strategies in a secondary school as: a theory, a culture of diversity, a cooperative team and support from administrative leaders.
\end{abstract}

Key words: Adaptive Education Strategies. Secondary Education. Feasibility.

* Profesora Titular de Pedagogía Diferencial en el Departamento de Métodos de Investigación y Diagnóstico en Educación de la Universidad Complutense de Madrid. Sus líneas de investigación se centran en las áreas de Educación Infantil (evaluación de programas; modelo de calidad) y en Educación Adaptativa (desarrollo y evaluación de estrategias de adaptación; diferencias individuales relacionadas con el aprendizaje; teoría educativa diferencial). 


\section{Introducción}

El concepto de adaptación no es nuevo ni en la teoría educativa ni en la legislación en materia de educación ni, por supuesto, en la práctica escolar. De hecho las adaptaciones actuales contemplan gran parte de las realizadas con anterioridad, como son la diferenciación de instituciones o la de los niveles y ciclos educativos o la de los agrupamientos o, en definitiva, la individualización educativa (García, 1991). Tanto el agrupamiento como la individualización fueron formas de adaptación educativa que recibieron gran atención durante los años setenta y ochenta, diseñando programas, implementándolos y evaluándolos como respuestas educativas eficaces a la diversidad de la escuela. Tanto una como otra utilizaron distintos criterios de diferenciación, como la aptitud cognitiva, el ritmo de aprendizaje, los intereses o el estilo de aprendizaje, aunque utilizaron procedimientos distintos. Y tanto una como otra partieron de enfoques teóricos diferentes, que pudiéramos resumir en adaptaciones centradas en la materia o en adaptaciones centradas en el alumno. En el primer caso se pautaba el ritmo y secuencia de aprendizaje a través de programas prescriptivos para la consecución de objetivos previamente determinados. En el segundo caso, por el contrario, se diseñaban programas individuales en función de los objetivos de cada estudiante. La tendencia actual es la utilización de una y otra forma de adaptación para que, de este modo, confluyan las necesidades de la sociedad donde el estudiante deberá integrarse, las propiedades de las materias de enseñanza y las características propias del alumno en sus diferentes situaciones instructivas (Corno y Snow, 1986; García; 1995; Wang, 1995).

La reforma educativa del sistema español plantea el tema de la adaptación educativa proponiendo tres niveles de adaptación - al centro, al grupo y al estudiante individual- tanto para plantear objetivos específicos y significativos como para facilitar la consecución de dichos objetivos por parte de los estudiantes. El profesorado de todas las etapas educativas es consciente de la heterogeneidad de las aulas y de la necesidad de adaptar las intervenciones educativas, pero también reconoce la inseguridad en el cómo ponerlas en práctica.

Este artículo nace como resultado de una experiencia de atención a la diversidad realizada en un centro de Educación Secundaria de la periferia de Madrid, combinando la teoría y la práctica para un objetivo común: disminuir el alto porcentaje de suspensos en materias instrumentales mediante estrategias de adaptación curricular. La experiencia duró un curso escolar, tras el cual todos los implicados pensamos que el esfuerzo había merecido la pena puesto que se había logrado aumentar el número de alumnos que aprobaron en el tercer trimestre. Sin embargo, no se extendió más del curso escolar ni parece que, tampoco, se observaron cambios positivos (ni negativos) en el centro más allá de lo que enriqueciera individualmente a los profesores que participaron en la experiencia.

Las reflexiones sobre los problemas y las posibles razones de esta situación pueden ayudar a futuros implementadores de estrategias de adaptación y es esto lo que a continuación presentamos. El objetivo de este artículo es, por lo tanto, poner de manifiesto los elementos que pueden facilitar la puesta en práctica de estrategias educativas desarrolladas para que todos los estudiantes logren los objetivos planificados, fruto de la reflexión teórica y de la observación de la práctica en un centro educativo. Sin tales requisitos, pensamos que abordarlas no dejarán de ser esfuerzos loables pero puntuales.

El diseño e implementación de estrategias de adaptación debe realizarse desde un planteamiento global, abordado desde el proyecto educativo del centro bajo un propuesta de res- 
peto y apoyo de la diversidad. Pensamos que toda intervención educativa tiene tintes de adaptación, de hecho sólo tenemos que pensar en la edad de los estudiantes para constatar esta afirmación. Pero en este artículo no nos vamos a referir a esas adaptaciones, de carácter excesivamente amplio y poco individualizado ni a describir o justificar las estrategias de adaptación y diversidad curricular introducidas en la LOGSE ni tampoco se dirigirá a los alumnos con necesidades educativas especiales, sino a las estrategias que se deben abordar en el aula, en aquellas materias donde los estudiantes diariamente se enfrentan con nuevos problemas de aprendizaje y la forma en que podemos hacer frente a estas diferencias para ayudar técnicamente a superarlos. Sería retornar a los principios y modelos de individualización educativa pero en contextos de aula con las condiciones y recursos que nuestros centros de secundaria poseen. Hay que insistir en que muchas de las ideas que presentamos no son nuevas, el artículo tiene como objetivo el que reflexionemos precisamente basándonos en todo el bagaje pedagógico que tenemos, recogiendo esas experiencias probadas con éxito en contextos escolares y evitando caer en errores que otros han cometido o de los que ya nos han advertido.

\section{Delimitación conceptual: diversidad y atención a la diversidad}

En primer lugar es necesario que planteemos la forma que tenemos de entender la adaptación educativa, principalmente los conceptos de diversidad y atención a la diversidad o adaptación educativa, puesto que ello condiciona las reflexiones posteriores.

- Diversidad. ¿Somos todos iguales? ¿los alumnos? ¿los profesores? Es más ¿Todas las escuelas son iguales? ¿todas las materias? ¿todas las tareas de aprendizaje? ... aceptar las diferencias en cada sujeto y en cada situación educativa supone un punto de partida, necesario para enfrentarnos a las situaciones heterogéneas de las aulas y buscar respuestas educativas alternativas y ajustadas a las necesidades de los implicados. De esta manera, suponemos que la intervención será más eficaz y eficiente.

- Atención a la diversidad o adaptación educativa. Toda respuesta, tanto a largo como a corto plazo, incluso la respuesta inmediata durante el proceso instructivo, que se hace pensando en el logro de los objetivos y en el tipo de alumno son formas de atender o adaptarnos a la diversidad del estudiante. Si bien entendemos atención a la diversidad como adaptación educativa a las necesidades de todos los estudiantes, lo cierto es que en la mayoría de los casos esa atención se dirige a los alumnos con dificultades educativas, con problemas de aprendizaje o con una baja actitud hacia la escolaridad. Quizás por ser uno de los problemas que la escuela quiere solucionar con más urgencia. En Educación Secundaria Obligatoria y en determinados contextos ese problema se traduce en un alto porcentaje de alumnos que no consiguen los objetivos.

De todas formas la adaptación no debe ser una medida «extraordinaria», muy por el contrario, debe ser un procedimiento habitual de carácter preventivo más que correctivo. Si el punto de partida es que cada estudiante tiene necesidades distintas y utiliza procedimientos diferentes para aprender, entonces la tarea pedagógica será, precisamente, utilizar distintos medios, actividades, métodos en pro de la mejora escolar. El símil que aclara este apartado lo encontramos en el mundo médico: ¿cuántos antibióticos puede haber en el mercado? seguro que la respuesta es cientos y que nosotros, como pacientes, hemos tomado en algún 
momento alguno, incluso varios, diferentes en función del tipo de infección que hayamos tenido. El médico prescribe diferente antibiótico en función de la bacteria y del paciente. Y nosotros, ¿por qué no utilizamos también diferentes métodos, refuerzos, actividades... en función del objetivo y del alumno?

Corno y Snow (1986) entienden la educación como un programa de desarrollo de aptitudes, y hablan de dos formas de adaptación: el desarrollo directo y la circunvalación de ineptitudes. El primero supone buscar el programa o el método más adecuado en función de los objetivos a conseguir, pero la circunvalación de ineptitudes implica tener diferentes alternativas educativas que se ajusten a los diferentes estudiantes para conseguir el mismo objetivo. Mientras que el desarrollo de aptitudes se utiliza habitualmente, el segundo procedimiento no es frecuente en las escuelas.

Sin embargo, no todo debe ser adaptado. Hay áreas, actividades o tareas que todos los alumnos consiguen de forma eficaz o eficiente con el método utilizado $\mathrm{y}$, en consecuencia, ésta debe ser la forma de proceder. También pudiera ser que lo deseable sea provocar la modificación de las estrategias que utiliza habitualmente el estudiante porque su logro sería un resultado deseable, bien porque pueda ser transferida a otras situaciones, bien porque sean parte de un desarrollo integral. Por ejemplo, parece que el estudiante independiente de campo prefiere y le beneficia el trabajo individual más que el trabajo en grupo. Pero los planificadores educativos estamos de acuerdo en que uno de los objetivos educativos comunes es el de ser capaz de trabajar en equipo, entonces una forma de hacerlo es provocar este tipo de situaciones, adecuadamente secuenciadas y dirigidas, para que resulten productivas y sea una forma de entrenar las destrezas implicadas. Entonces, ¿cuándo debemos plantearnos la adaptación educativa? Creo que es aconsejable en cuatro casos:

1. En áreas que tradicionalmente sean problemáticas. Es decir, allí donde es habitual que suspenda un alto porcentaje de alumnos o, incluso, que más de la mitad sólo logren aprobar, no dominar el contenido de aprendizaje. El concepto de dominio, importante que fue para los sistemas de individualización educativa, debe ser retomado y empleado sistemáticamente en la escuela (Bloom, 1976).

2. En los objetivos que todos los alumnos deban dominar por ser conocimientos, actitudes, destrezas, procedimientos... básicos para aprendizajes posteriores o transferibles a otras situaciones (escolares, familiares, profesionales, etc.) como son, por ejemplo, la comprensión lectora o la autoestima.

3. En aquellos campos donde puedan ser ofrecidas alternativas vinculadas a los intereses o potencialidades de los estudiantes, para promover el desarrollo máximo personal. Es decir, es importante que todos los estudiantes consigan los objetivos básicos planificados, pero también que la educación pueda ser diferenciadora, potenciando la individualidad. Lo que supondrá incorporar programas diferentes para los escolares, programas de desarrollo musical, programas de matemáticas avanzadas, etc.

4. En situaciones donde existan dos ó más métodos eficaces y cada uno exija estrategias cognitivas o habilidades diferentes en los alumnos. Por ejemplo, en el aprendizaje de la lectura, aprendizaje básico al iniciar Primaria, y que en más o menos tiempo todos los niños llegan a dominar al acabar el primer ciclo. ¿Alguna vez nos hemos planteado si aquellos que tardan más o que, objetivamente, parece que les cuesta o aburre dicho aprendizaje, no sea porque estemos utilizando el método menos ajustado para ellos? Como al final los niños terminan leyendo, suponemos que el 
método que hemos utilizado ha sido eficaz, sin embargo, estamos siendo menos eficientes, puesto que el coste de la enseñanza no sólo se mide en términos económicos sino en coste de esfuerzo y perjuicio / beneficio de las aptitudes y actitudes que provoca en el aprendizaje, actitud, autoconcepto... del estudiante.

\section{Breve descripción de la experiencia de atención a la diversidad en un centro de ESO}

El IES estaba situado en un barrio de Madrid con una situación económico deteriorada y que recibe alumnos que, en general, tienen un historial de dificultades de aprendizaje. Una de las preocupaciones del profesorado de ESO es el elevado porcentaje de alumnos que no consiguen los objetivos y contenidos mínimos para esta etapa, además se diagnostica una actitud negativa hacia las tareas de aprendizaje, una motivación de logro externa y poco controlable y deficitarios hábitos de estudio. Este conjunto de características pueden ser atendidas educativamente $y$, en consecuencia, corregir o mejorar. Pero una respuesta de este tipo conlleva una adaptación global del currículum que no sólo repercute en modificaciones puntuales de estrategias didácticas o materiales instructivos sino que también plantea modificaciones en objetivos, en el ritmo y en la secuencia del aprendizaje. El problema, en la práctica, era que ello suponía un cambio global en la organización del centro así como una mayor dotación de recursos materiales y, sobre todo, personales, asunto de difícil solución. Luego, para esta experiencia, se decidió por introducir estrategias más viables que afectaran sólo al aula, en tres grupos de $3^{\circ}$ de ESO, en tres áreas de conocimiento (Biología, Física y Química y Lengua) en función del grado de implicación del equipo docente, y en las unidades instructivas programas para el tercer trimestre del curso escolar.

Para responder mejor a las características de los alumnos de estos grupos, definidos como alumnos de baja aptitud en el enfoque adaptativo, las estrategias formuladas se basaron en las del Mastery Learning (Bloom, 1976; Anderson y Block, 1985; González, 1991). Así, aunque el grupo era la unidad básica de trabajo, predominaba el trabajo individual de los alumnos durante las actividades de aprendizaje. Estas estrategias supusieron un cambio en la organización del aula (trabajo individual o de pequeño grupo más que de gran grupo con los materiales diseñados); en el rol del profesor (invirtiendo más tiempo en la planificación de la enseñanza y en el seguimiento del aprendizaje individual que en la enseñanza expositiva y grupal); en el rol del alumno (que pasa más tiempo realizando actividades guiadas de forma escrita que escuchando explicaciones); en el material didáctico (de uso individual, construido previa y específicamente para las unidades programadas en función de los objetivos y contenidos básicos de las unidades didácticas y organizados secuencialmente en función de la estructura de cada materia) y en el ritmo de enseñanza (individual durante el tiempo establecido para la unidad).

Al mismo tiempo se observaron diferencias en las características de los alumnos y en las características de los contenidos de las áreas de conocimiento a intervenir, por lo que las estrategias generales se concretaron para cada área. En el área de Lengua, los contenidos no eran nuevos para los alumnos, de una forma u otra ya habían sido trabajados en cursos anteriores aunque con diferente nivel. En el diagnóstico se manifestaron diferencias importantes en el nivel inicial de los alumnos por lo que se decidió reforzar contenidos deficitarios y desarrollar unidades previas a las planificadas para el trimestre, junto con unidades de re- 
fuerzo complementarias (comprensión lectora, vocabulario, ortografía, etc.). El pretest sirvió para situar al alumno en el nivel adecuado y prescribirle las unidades a trabajar. En definitiva, se utilizaron estrategias directivas diferenciadas por objetivos y contenidos.

En las áreas de Biología y Física/Química los contenidos no eran nuevos para los alumnos por lo que las diferencias iniciales se relacionaban más con la capacidad de asimilación y con el interés del alumno. Se optó, en consecuencia, por diseñar estrategias semi-directivas con materiales que desarrollaran los mismos objetivos pero que diferenciaran en dos niveles los contenidos procedimentales y el nivel de profundización de los conceptos trabajados. El alumno elegía el nivel de dificultad y el ritmo de trabajo deseado, pudiendo cambiar de nivel durante el proceso instructivo.

\section{Viabilidad, eficacia y problemas de las estrategias de adaptación educativa}

Demostrar que se pueden desarrollar estrategias de adaptación en entornos naturales y que, además, pueden ser eficaces, en términos operativos supone comprobar la viabilidad y la eficacia de las estrategias de adaptación que se diseñaron. La viabilidad se evaluó en función de la concordancia entre la implementación y el diseño del modelo de adaptación elegido. La eficacia se evaluó a través de la mejora del rendimiento, de las conductas de aprendizaje y de la satisfacción de alumnos y profesores con los resultados logrados.

Las estrategias de adaptación diseñadas para los alumnos del centro se juzgaron como viables, por dos razones principalmente: la primera, porque se desarrollaron y finalizaron en el tiempo previsto de intervención y con los recursos habituales del centro, sin implicar a ningún profesor ni servicio desinteresado; en segundo lugar, porque las modificaciones que hubo que introducir no afectaron al modelo en sí, sino a elementos puntuales vinculados, básicamente, a la falta de recursos (temporales, espaciales, personales y materiales).

Si juzgamos la fidelidad de la implementación de las estrategias de adaptación con el diseño, los elementos más fáciles de implementar fueron la estructura y secuencia del proceso de enseñanza-aprendizaje planificados así como la asignación de materiales a los alumnos. Por el contrario, los elementos más difíciles se refirieron a la organización del aula, al rol del profesor y a la evaluación, que sufrieron cambios durante la intervención.

Por otro lado, las estrategias de adaptación se valoraron como eficaces tanto en la vertiente de los resultados logrados (rendimiento, actitud positiva, interés e implicación en el proceso de aprendizaje, ...) como en la satisfacción de los profesores y de los alumnos con las estrategias utilizadas como en la percepción del profesorado sobre la mejora de su competencia docente (Castro et al., 1996; García et al., 1996).

$\mathrm{Si}$, como estamos diciendo, las estrategias de adaptación que se diseñaron para esa situación resultaron relativamente viables y eficaces, entonces ¿dónde está el problema? El problema principal es que las estrategias finalizaron con el proyecto que motivó su desarrollo. Algunas de las razones tan simples como: fin de los recursos económicos puesto que no hubo ninguna convocatoria de ayuda posterior que permitiera continuar al equipo de investigación; el desentendimiento del centro y, más concretamente, del departamento de orientación y de alguno de los departamentos de área que colaboró; y la permanencia en el centro de tal sólo un docente de un total de cuatro que iniciaron el proyecto. 


\section{De la experiencia a la reflexión: orientaciones para llevar a cabo estrategias de adaptación}

Los puntos fuertes de nuestra experiencia habría que centrarlos en la formulación de un plan integral con sustento teórico, la utilización de las clásicas estrategias de individualización educativa, la formación de un equipo cooperativo e interdisciplinar de investigación con profesores universitarios y profesores de secundaria, el diagnóstico situacional y un plan sistemático de evaluación, la elaboración de materiales curriculares específicos para los objetivos del trimestre en las áreas a intervenir o a reforzar.

Por el contrario, se puso en evidencia que tan necesario como invertir esfuerzo, tiempo y recursos, es plantearse la implicación en una cultura de atención a la diversidad plasmada en un proyecto de la comunidad educativa, lo que permitiría una organización flexible, la racionalización de funciones y recursos, la exigencia de apoyos externos, etc. Aspectos en los que el Departamento de Orientación debería de tener una función clave como dinamizador, coordinador, diseñador, gestor y evaluador.

Enunciaremos y justificaremos, a continuación, los elementos que nos parecen fundamentales a la hora de abordar la atención a la diversidad en los centros de secundaria:

\section{EI marco teórico}

El hacer planteamientos coherentes de atención a la diversidad supone elaborar y discutir el marco en el que se diseñará la práctica y se introducirán las modificaciones pertinentes tras la evaluación procesual y final.

El marco que a nosotros nos orienta es el enfoque denominado Educación Adaptativa, derivado de los planteamientos de Snow (Cronbach y Snow, 1977; Snow y Lohman, 1984; Corno y Snow, 1986; Snow, 1997), Glaser (1977, 1988) y Wang (1995) y que hemos presentado más extensamente en otros trabajos (García, 1991, 1997), junto con los resultados de la investigación evaluativa de estrategias educativas desarrolladas para atender de algún modo la diversidad de las aulas, como el agrupamiento, la individualización o la enseñanza cooperativa.

La Educación Adaptativa explica el éxito, o fracaso escolar, por la interacción estudiante-situación instructiva, es decir, el que un estudiante consiga los resultados esperados no sólo depende de sus características personales, ni tampoco exclusivamente del método de enseñanza utilizado, sino del grado de ajuste del método al estudiante particular. Por consiguiente, lograr los resultados esperados depende de la inclusión de cuatro elementos:

a) la situación instructiva particular,

b) el tipo de objetivo,

c) el perfil individual del estudiante y

d) el ajuste de la enseñanza al perfil individual.

A partir de esta información se deben discutir y consensuar los principios de adaptación educativa, más las estrategias de adaptación en el aula, más la organización y recursos del centro y del aula, más el rol del profesor y del alumno... 
Pensamos que compartir un modelo teórico no sólo repercute en la asunción de métodos y roles coherentes sino también en la elaboración de actividades, materiales y estrategias más variadas y significativas para el aprendizaje, sirve de formación docente centrada en su práctica, y da la posibilidad de valorar los resultados y tomar decisiones de mejora, si ha lugar, en función de los principios que guiaron la intervención. En cualquier caso, supone un proceso de reflexión y de cambio escolar.

Como ejemplo presentamos los principios formulados en nuestra experiencia:

\section{PRINCIPIOS DE ADAPTACIÓN EDUCATIVA}

I. Cada clase tiene alumnos con intereses, niveles de conocimiento y necesidades individuales que aprenden de forma diferente y a distinto ritmo.

II. El alumno debe sentirse protagonista de la intervención, comprometido con su aprendizaje y capaz de lograr los resultados esperados.

III. La enseñanza debe proporcionar suficiente estructura a los alumnos con dificultades o baja motivación para el aprendizaje de forma que se les garantice éxito en un breve plazo y la recuperación de lo no aprendido, al mismo tiempo que permite ampliar el conocimiento a los alumnos más rápidos.

IV. Los contenidos de aprendizaje deben partir y ajustarse al nivel de conocimiento e intereses de los alumnos, aunque sin perder de vista los objetivos formulados en la programación del nivel educativo.

V. Cada materia tiene características diferentes que deben tenerse en cuenta a la hora de seleccionar las estrategias educativas y las formas de adaptación más adecuadas.

VI. La intervención educativa debe basarse en el dominio de los conceptos y destrezas básicas (formulados en los objetivos), respetando el ritmo de aprendizaje de los alumnos. Además debe permitir el seguimiento constante del aprendizaje de los alumnos, la detección y corrección inmediata de los errores, y una mayor cantidad de práctica de los conceptos difíciles o nuevos ajustada a las propias necesidades de cada alumno. Asimismo, debería incluir experiencias de aprendizaje que requieran la iniciativa y exploración del alumno, y que permitan la cooperación entre compañeros.

VII. El eje directriz de la intervención son los objetivos de las unidades instructivas. En cada unidad se deben formular y secuenciar los objetivos, diseñarse actividades de aprendizaje para distintos niveles iniciales que permitan el trabajo individual y la ejercitación de los conceptos y destrezas básicos. Además, en cada unidad se planificarán actividades de refuerzo para los alumnos más necesitados y de ampliación para los alumnos que consigan antes que sus compañeros los objetivos de la unidad. Asimismo, se elaborarán previamente los materiales de aprendizaje y las pruebas de evaluación.

VIII. Las estrategias educativas se deberán centrar en las necesidades del alumno y en el dominio de los objetivos de las unidades planificadas. Ello supone un ciclo educativo que se inicia con el diagnóstico del nivel de conocimientos de los alumnos en los objetivos programados y finaliza con la evaluación de dichos objetivos y toma de decisiones ajustadas a los resultados obtenidos con el fin de asegurar el dominio de los objetivos por todos los alumnos antes de pasar a los de unidades posteriores. 


\section{La cultura de la atención a la diversidad}

Cuantas veces hemos oído en los cursos impartidos: eso está muy bien en teoría, pero es imposible en la escuela. En cambio, estamos convencidos de que es posible pero sólo con una actitud, disposición y cooperación de un equipo docente implicado y lógicamente con el respaldo de los servicios técnico-administrativos, es decir, del Consejo Escolar, de la Dirección del centro y del Departamento de Orientación, pero también de la Inspección Educativa, de los CPRs, de los departamentos universitarios de las Facultades de Educación, incluso de la Administración Educativa en forma de financiación y normativa que facilite, por ejemplo, la permanencia del profesorado en los centros que inician este tipo de proyectos.

Nuestra experiencia nos puso en evidencia que la implicación del centro, en su conjunto, en un proyecto de atención a la diversidad y, sobre todo, la implicación del profesorado en la elaboración, desarrollo y evaluación de tal proyecto, puede relacionarse con el grado de coherencia entre el diseño de las estrategias de adaptación y su implementación, y es factor clave de la viabilidad de las adaptaciones. Todo ello tiene que ver con la aceptación de la diversidad de los estudiantes y un plan sistemático de respuesta educativa en el que estén de acuerdo e implicados todos los sectores del centro, en definitiva, una cultura de respeto y atención a la diversidad de todos los estudiantes.

$\mathrm{Si}$ en el centro no existe una cultura de atención a la diversidad o hay sectores reacios a su implantación, no debe abordarse de forma global un sistema de adaptación. Por el contrario, debe plantearse por etapas, es decir empezar por situaciones particulares y controlables e ir ampliándolas progresivamente.

\section{El diagnóstico de la diversidad}

Cada centro tiene su particularidad, luego igual que una buena intervención empieza por un buen diagnóstico, las adaptaciones que planifiquemos tendrán éxito sólo en la medida que conozcamos la situación del centro, sus potencialidades y necesidades. El análisis del contexto escolar supone:

1. Describir las características del centro (tipo de enseñanzas impartidas; recursos personales, materiales, económicos; infraestructura de la zona; cultura hacia la diversidad). Una vez conocido se pueden tomar decisiones, en primer lugar, de si debe emprenderse algún plan de atención a la diversidad y, en caso positivo, en qué ciclos intervenir, en qué materias, con qué grupo de profesores, de qué forma y con qué recursos.

2. Analizar diferencialmente a los alumnos en cuanto a resultados y áreas de éxito/fracaso y perfiles aptitudinales relacionados con cada área. En nuestro estudio, los factores más relacionados con el bajo rendimiento no siempre fueron las bajas capacidades; además se puso en evidencia, como era de esperar según la teoría que dirigió nuestro trabajo, que cada área de conocimiento tenía su propio grupo de aptitudes. El número de horas dedicadas al estudio y el rendimiento previo en esa materia concretamente podrían considerarse como las características comunes en todas las áreas, aptitudes que se combinan diferencialmente según las materias (por ejemplo, con razonamiento e importancia que el alumno concede a esa materia en Ciencias Naturales; con factor verbal y la percepción de los padres sobre su hijo como estudiante en Lengua y Literatura Castellana; etc. (Castro et al. 1996). 
Un diagnóstico diferencial que identifique las fuentes relacionadas con un buen o mal resultado educativo en cada área de conocimiento, permite determinar el tipo de intervención más adecuada para cada tipo de estudiante en cada materia (adaptación al nivel de aprendizaje, refuerzo previo de aprendizajes básicos, tiempo instructivo, diferenciación de actividades y materiales, etc.)

3. Análisis de los objetivos, contenidos, materiales instructivos y criterios de evaluación de las áreas en las que se decida intervenir de forma adaptada. La finalidad es identificar los objetivos más importantes y los complementarios, seleccionar y diseñar actividades diferentes para un mismo objetivo y elaborar pruebas que determinen el dominio de las unidades instructivas, tal como proponían las estrategias de mastery learning (Anderson y Block, 1985).

4. Análisis de las adaptaciones realizadas por los profesores: experiencias de adaptación; tipo de adaptaciones y elementos curriculares adaptados; tipo de alumnos para los que se prevén las adaptaciones; quiénes y con qué recursos se desarrollan; valoración de las adaptaciones realizadas. Como todos los centros se ha planteado en algún momento algún tipo de adaptación, es importante valorar aquéllas que el profesor aborda en el aula. Quizás no lo haga de forma sistemática, pero es importante anotar aquéllas que les resultan eficaces en determinadas situaciones y para algún tipo de estudiante en particular. Y, en consecuencia, evitar o utilizar estrategias en función de dicha práctica.

\section{Un trabajo cooperativo y continuado, coordinado por el Dpto. de Orientación}

La adaptación educativa va unida a la flexibilización de espacios, de tiempos, de recursos, de grupos..., por lo que en el centro se van a producir algunos cambios. Pero los cambios no son positivos por sí mismos, sólo bajo una propuesta coherente de atención a la diversidad, puede ser pertinente y eficaz. Y esta propuesta supone un trabajo previo de preparación y planificación, un seguimiento de control y ajuste y una evaluación con vistas a mejorar para poder continuar con lo empezado. Supone la utilización de un trabajo interdisciplinar, coordinado desde el departamento de Orientación del centro, puesto que se necesitan especialistas en: programas educativos, diagnóstico, diseño educativo, procedimientos de evaluación a la vez que especialistas curriculares en las áreas a intervenir con algún año de experiencia de enseñanza y observador del aprendizaje.

Este trabajo requiere continuidad y un plan a medio y largo plazo que debe iniciarse antes del curso, incluso dedicar un año con reuniones periódicas antes de empezar la intervención. Reuniones que deben prolongarse durante el año que se inicie el programa, semanal o quincenalmente, para revisar el plan y tomar nota de los cambios y modificaciones introducidos. Trabajo que debe evaluarse sistemáticamente también al final del mismo, con sesiones intensivas para determinar la eficacia y tomar decisiones sobre los cambios a introducir y prepararlo para el siguiente curso.

\section{Un apoyo de los responsables de la administración educativa}

La atención a la diversidad en los centros educativos supone una implicación y apoyo del centro en su conjunto, incluso de aquellos que no quieran o no crean en este tipo de es- 
trategias. Porque el centro debe reorganizar el horario, y los profesores estar de acuerdo, para que los docentes implicados puedan estar en los mismos equipos didácticos, en los cursos y materias asignados para intervenir y dispongan, junto al pedagogo o psicopedagogo del departamento de orientación, de tiempos y espacios comunes de trabajo conjunto. Y cuando se dice el centro, también el apoyo e incluso el reconocimiento y valoración de la administración educativa, a través de la inspección y de los centros de formación del profesorado.

Facilitar la adaptación también es procurar un mayor tipo de recursos, quizás no en cantidad sino en utilización. Sobre todo en lo que se refiere a las aulas y las materias objeto de intervención. En un sistema individualizado, la información y explicaciones del profesor a todo el grupo disminuye para aumentar las tareas de supervisión, orientación individual y evaluación de las actividades de aprendizaje. El profesor, mientras los alumnos trabajan en sus materiales, se mueve por el aula e interactúa con los estudiantes para seguirles en su trabajo y progreso (además de su implicación en el diseño y evaluación del programa). Cuando prevalezca este tipo de trabajo individual de los alumnos, no sólo es aconsejable, es necesario incorporar un ayudante (persona en prácticas o un profesor liberado del equipo docente) para poder atender a cada estudiante adecuadamente y en el momento que lo precise. Pero para que su labor sea eficaz, tendrá que ser una persona preparada en la materia y en la metodología utilizada, coordinada y supervisada por el profesor titular y con funciones claramente delimitadas.

En cuanto a los recursos, es importante diseñar, adquirir o seleccionar materiales adecuados, a las necesidades de las unidades instructivas y a las características de los alumnos, y variados para el mismo tipo de objetivo. Una vez más la implicación del profesor de estas materias es fundamental para diseñar o analizar y clasificar materiales diversificados. En nuestro caso, fueron precisamente los materiales diseñados por los profesores del equipo de investigación para sus materias los más ajustados y los únicos que después han seguido utilizándose; pero también se puso en evidencia la necesidad de recomendaciones técnicas para elaborarlos y, algo más difícil pero recomendable, encontrar editoriales que estén dispuestas a elaborar materiales variados partiendo de las necesidades de cada centro.

\section{Consideraciones finales}

Las estrategias de adaptación adaptativa pueden ser un medio de iniciar un proyecto común en los centros de secundaria que, directa e indirectamente repercutiría tanto en la mejora de los resultados educativos de todos los alumnos como en la cultura educativa del centro. La aceptación y respeto de la diversidad de los centros es un punto de partida para la reflexión, debate y búsqueda de soluciones viables que comprometan a todos los implicados.

Sin embargo, las estrategias de adaptación sólo serán válidas y eficaces en la medida que se inviertan esfuerzo, tiempo y recursos a medio y largo plazo y que se logre un apoyo interno y externo que fomente las condiciones que favorecen el diseño, desarrollo y evaluación de este tipo de proyectos.

En Educación Secundaria nos encontramos con muchos profesores especialistas en las áreas curriculares pero con escasa formación pedagógica o psicopedagógica. Por eso se hace tan importante que en el Departamento de Orientación del centro se asuma ese papel 
técnico que guíe, supervise y controle los procesos de atención a la diversidad en la línea que hemos indicado en este artículo.

\section{Referencias bibliográficas}

Anderson, L. W. y Block, J. H. (1985): Mastery Learning Model of Teaching and Learning. En T. Husen y T. N. Postlethwaite (eds.). The international Enciclopedia of Education. Oxford: Pergamon, 3219-3230.

Bloom, B. S. (1976): Human characteristics and school learning. New York: McGraw Hill.

Castro, M., García, M., Monge, C., Martínez, J., Asensio, I, López, E., Ruiz, C., Carlero, J. M. y Juiz, M. T. (1996): Bajo rendimiento y atención a la diversidad en Educación Secundaria Obligatoria: Estudio de los factores determinantes del bajo rendimiento y de las respuestas educativas para el alumnado que no alcanza los objetivos mínimos y propuestas de mejora. Informe. Convocatoria MEC de Proyectos de cooperación de Institutos de Educación Secundaria y Departamentos Universitarios, convocatoria 1995. Madrid: MEC

Corno, L. y Snow, R. E. (1986): Adapting teaching to individual differences among learners. En C. M. Wittrock (ed.). Handbook of research on teaching. London: MacMillan, 605-629.

Cronbach, L. J. y Snow, R. E. (1977): Aptitudes and instructional methods. A handbook for research on interactions. New York: Irvington Publishers.

García, M. (1991): Proyecto Docente de Pedagogía Diferencial. Dpto. M.I.D.E. Universidad Complutense, Madrid. Doc. no publicado.

García, M. et al (1996): Propuestas de adaptación curricular para la educación secundaria obligatoria: experiencia en el IES P-V de Madrid. Comunicación presentada al XI Congreso Nacional de Pedagogía. S. Sebastián.

García, M., Mafokozi, J. y Martin, C. (1995): La adaptación educativa a las diferencias individuales en los últimos cinco años (1990-1995). En AIDIPE (comp.). Estudios de investigación educativa en intervención psicopedagógica: Valencia, 91-94.

García, M. (1997): Educación Adaptativa. En Revista de Investigación Educativa, 15 (2), 247-271

Glasser, R. (1977): Adaptive Education: Individual diversity and learning. New York: Holt, Rinehart y Winston.

GLASSER, R. (1988): General discussion: relationships between aptitud, learning and instruction. En R. E. Snow, P. A. Federico y W.E. Montagne (eds.). Aptitude, learning and instruction. Hillsdale, New Jersey: LEA, vol. 2, 209-326.

González Galán, M. A. (1991): La enseñanza individualizada y los procedimientos de aprendizaje para el dominio. En C. Jiménez (coord.). Lecturas de Pedagogía Diferencial. Madrid: Dykinson, 239-258.

Snow, R. E. (1997): Aptitudes and symbol systems in Adaptive Classroom Teaching. En Phi Delta Kappan, 1, 354-360

Snow, R. E. y Lohman, D. F. (1984): Toward a theory of cognitive aptitude for learning from instruction. En Journal of Educational Psychology, 76, 347-376.

Wang, M. C. (1995): Atención a la diversidad del alumno. Madrid: Narcea.

Fecha de recepción: 10-11-00

Fecha de revisión: 18-11-00

Fecha de aceptación: 21-11-00 